\title{
Expression of sclerostin in the regenerating scales of goldfish and its increase under microgravity during space flight
}

Tatsuki Yamamoto ${ }^{1 *}$, Mika Ikegame ${ }^{2 *}$, Jun Hirayama ${ }^{3}$, Kei-ichiro Kitamura ${ }^{4}$, Yoshiaki Tabuchi ${ }^{5}$, Yukihiro Furusawa $^{6}$, Toshio Sekiguchi ${ }^{1}$, Masato Endo ${ }^{7}$, Hiroyuki Mishima ${ }^{8}$, Azusa Seki ${ }^{9}$, Sachiko Yano ${ }^{10}$, Hajime Matsubara $^{11}$, Atsuhiko HatTori ${ }^{12}$, and Nobuo SuZukI ${ }^{1}$

${ }^{1}$ Noto Marine Laboratory, Division of Marine Environmental Studies, Institute of Nature and Environmental Technology, Kanazawa University, Noto-cho, Ishikawa 927-0553, Japan; ${ }^{2}$ Department of Oral Morphology, Okayama University Graduate School of Medicine, Dentistry and Pharmaceutical Sciences, Okayama, Okayama 700-8525, Japan; ${ }^{3}$ Department of Clinical Engineering, Faculty of Health Sciences, Komatsu University, Komatsu, Ishikawa 923-0961, Japan; ${ }^{4}$ Department of Clinical Laboratory Science, Division of Health Sciences, Graduate School of Medical Science, Kanazawa University, Kodatsuno, Ishikawa 920-0942, Japan; ${ }^{5}$ Life Science Research Center, University of Toyama, Sugitani, Toyama 930-0194, Japan; ${ }^{6}$ Department of Liberal Arts and Sciences, Toyama Prefectural University, Kurokawa, Toyama 939-0398; ${ }^{7}$ Department of Marine Biosciences, Tokyo University of Marine Science and Technology, Minato-ku, Tokyo 108-8477, Japan; ${ }^{8}$ Department of Dental Engineering, Tsurumi University School of Dental Medicine, Yokohama, Kanagawa 2308501, Japan; ${ }^{9}$ HAMRI Co. Ltd., Koga, Ibaraki 306-0101, Japan; ${ }^{10}$ Japan Aerospace Exploration Agency, Tsukuba, Ibaraki 305-8505, Japan; ${ }^{11}$ Noto Center for Fisheries Science and Technology, Kanazawa University, Ossaka, Noto-cho, Ishikawa 927-0552, Japan; ${ }^{12}$ Department of Biology, College of Liberal Arts and Sciences, Tokyo Medical and Dental University, Ichikawa, Chiba 272-0827, Japan

(Received 21 September 2020; and accepted 4 October 2020)

\begin{abstract}
Osteocytes, osteoblasts (bone-forming cells), and osteoclasts (bone-resorbing cells) are the primary types of cells that regulate bone metabolism in mammals. Sclerostin produced in bone cells activates osteoclasts, inhibiting bone formation; excess production of sclerostin, therefore, leads to the loss of bone mass. Fish scales have been reported to have morphological and functional similarities to mammalian bones, making them a useful experimental system for analyzing vertebrate bone metabolism in vitro. However, whether fish scales contain cells producing sclerostin and/or osteocytes has not been determined. The current study demonstrated, for the first time, that sclerostin-containing cells exist in goldfish scales. Analysis of the distribution and shape of sclerostin-expressing cells provided evidence that osteoblasts produce sclerostin in goldfish scales. Furthermore, our results found that osteocyte-like cells exist in goldfish scales, which also produce sclerostin. Finally, we demonstrated that microgravity in outer space increased the level of sclerostin in the scales of goldfish, a finding suggesting that the induction of sclerostin is the mechanism underlying the activation of osteoclasts under microgravity.
\end{abstract}

Address correspondence to: Nobuo Suzuki, Noto Marine Laboratory, Division of Marine Environmental Studies, Institute of Nature and Environmental Technology, Kanazawa University, Noto-cho, Ishikawa 927-0553, Japan

Tel: +81-768-74-1151, Fax: +81-768-74-1644

E-mail: nobuos@staff.kanazawa-u.ac.jp

\section{INTRODUCTION}

Osteoblasts and osteoclasts are the two primary cell types that regulate bone metabolism. Osteoblasts are responsible for the formation of new bone, while osteoclasts are involved in the resorption of aged bone (Henry and Bordoni 2020). Sclerostin, a protein encoded by the Sost gene, is a negative regulator of

\footnotetext{
*These authors equally contributed to this study.
} 
bone formation. Mutations in Sost are associated with bone diseases characterized by high bone mass, such as sclerosteosis and Van Buchem disease (Balemans et al. 2001; Brunkow et al. 2001; Balemans et al. 2002; Staehling-Hampton et al. 2002). In bone, Sost is primarily expressed in osteocytes; however, osteoblasts and osteoclasts have been found to have low levels of Sost (Collette et al. 2013). At the molecular level, sclerostin bounded to LRP5/6 receptors inhibited the $\mathrm{Wnt} / \beta$-catenin signaling pathway and then decreased bone formation ( $\mathrm{Li}$ et al. 2005; Poole et al. 2005; Ellies et al. 2006). It has been reported that both $\mathrm{Sost}^{-/-}$and osteocyte-ablated mice showed resistance to bone loss induced by hindlimb unloading via tail suspension (Tatsumi et al. 2007; Lin et al. 2009). These previous studies have provided evidence that sclerostin produced by osteocyte has an important role in the response of mechanical stimuli (Pajevic et al. 2013; DelgadoCalle et al. 2017; Hinton et al. 2018).

Osteoblastic cells regulate the differentiation and activation of osteoclasts in mammalian bone (Kondo et al. 2001; Kearns et al. 2008; Lacey et al. 2012). Some stromal cells, such as osteoblasts and bone marrow cells, produce the receptor activator for nuclear factor $\kappa \mathrm{B}$ ligand (RANKL). RANKL binds to the receptor activator for nuclear factor $\kappa \mathrm{B}$ (RANK) on osteoclasts and promotes osteoclast differentiation and activation (Kondo et al. 2001; Kearns et al. 2008). The effect of RANKL on osteoclasts is inhibited by osteoprotegerin (OPG), a decoy receptor produced in stromal cells. Mammalian sclerostin suppresses OPG production by inhibiting Wnt signaling in stromal cells, thus facilitating osteoclastogenesis (Kubota et al. 2009; Silva and Branco 2011; Pajevic et al. 2013; Delgado-Calle et al. 2017).

Several groups, including ours, have reported that fish scales are morphologically and functionally similar to mammalian bones in some ways. Fish scales contain osteoblasts, osteoclasts, and mineralized matrix (osseous layer, on epithelial side) underlined with collagen rich matrix (fibrous layer, on dermis side) (Bereiter-Hahn and Zylberberg 1993; Azuma et al. 2007; Ohira et al. 2007; Suzuki et al. 2007; Suzuki et al. 2008b; de Vrieze et al. 2014). We established fish scale as a useful experimental system for analyzing vertebrate bone metabolism in vitro (Suzuki et al. 2000; Suzuki and Hattori 2002). In this system, the expression of genes involved in bone metabolism and the activities of osteoblasts or osteoclasts show high sensitivity to a variety of mechanical stimuli, including static hypergravity by centrifugation (Suzuki et al. 2008a), dynamic hyper- gravity by vibration (Suzuki et al. 2007; Suzuki et al. 2009), ultrasound (Kitamura et al. 2010; Suzuki et al. 2016; Hanmoto et al. 2017), and microgravity in outer space (Ikegame et al. 2019). These responses provide evidence that fish scale is a suitable experimental model for analyzing the responses of bone metabolism to mechanical stimuli.

The current study demonstrated for the first time that sclerostin is produced in goldfish scales at the mRNA and protein levels. We also found evidence that osteocyte-like cells in a calcified matrix and osteoblasts in scales are responsible for sclerostin production. Finally, we found that microgravity in outer space increased the expression of Sost in scales, a possible mechanism of osteoclast activation.

\section{MATERIALS AND METHODS}

Animals. One female and one male goldfish (Carassius auratus) were purchased from Higashikawa Fish Farm (Yamatokoriyama, Japan). Artificial fertilization was carried out at Tokyo University of Marine Science and Technology to obtain fertilized eggs. The eggs were grown to adult fish with body lengths of $12-15 \mathrm{~cm}$. Then, adult fish were transferred to Kanazawa University and the Tokyo Medical and Dental University and used for in vitro experiments. Fish were fed a commercial pellet diet for puffer fish every morning and were maintained in fresh water at $26^{\circ} \mathrm{C}$ until used. All experimental procedures were conducted in accordance with the Guide for the Care and Use of Laboratory Animals prepared by Kanazawa University and the Tokyo Medical and Dental University.

Preparation of regenerating goldfish scales. Fully grown scales were removed from the goldfish, which were anesthetized with $0.03 \%$ ethyl 3-aminobenzoate methanesulfonic acid salt (Sigma-Aldrich, Inc., St. Louis, MO, USA), to allow for the regeneration of scales. On day 15 post removal, the regenerating scales were removed from the goldfish and were used for the experiments.

In situ hybridization. Digoxigenin (DIG)-labeled sense and antisense single-stranded RNA probes for goldfish Sost were prepared using DIG RNA Labeling Kits (Roche, Basel, Switzerland) in accordance with the manufacturer's instructions. The cDNA sequence used for the preparation of probes was a 293 bp fragment (AB970730).

The regenerating scales were fixed in 4\% paraformaldehyde (PFA) solution in phosphate buffered sa- 
line (PBS). After rinsing in RNase-free PBS, the scales were used as whole-mount samples or embedded in Tissue-Tek ${ }^{\circledR}$ Optimal Cutting Temperature Compound for the preparation of cryosections. Whole-mount and cryosection samples were pretreated with $0.2 \mathrm{~N} \mathrm{HCl}$, proteinase $\mathrm{K}$, and $0.25 \%$ acetic anhydride. The samples were then hybridized with the probes at a final concentration of $1 \mu \mathrm{g} / \mathrm{mL}$ in a hybridization solution composed of $50 \%$ deionized formamide, $2 \%$ blocking reagent, $5 \times$ saline-sodium citrate (SSC), $0.02 \%$ SDS, $0.1 \%$ N-lauroylsarcosine, and $200 \mu \mathrm{g} / \mathrm{mL}$ tRNA at $55^{\circ} \mathrm{C}$ overnight. After hybridization, the sections were washed with $0.1 \times$ SSC and $2 \times$ SSC and treated with ribonuclease. The probe signals were detected with anti-DIG antibody conjugated to alkaline phosphatase in accordance with the manufacturer's protocol (DIG Detection Kit; Boehringer Mannheim, Mannheim, Germany). The samples were counterstained with methyl green to visualize the nuclei and examined by light microscopy.

Immunohistochemistry. The rabbit anti-human sclerostin (N-terminal 12-42) (ab63097; Abcam, Cambridge, UK) was used as the primary antibody. The regenerating scales were fixed in 4\% PFA solution in PBS, and whole-mount or cryosection samples of scales were prepared as described above. The samples were incubated in blocking solution containing $0.1 \%$ Tween $20,0.3 \%$ glycine, $10 \%$ normal goat serum, and $1 \%$ bovine serum albumin (BSA) for $1 \mathrm{~h}$ at room temperature. Subsequently, they were incubated with the primary antibody $(\times 100)$ overnight at $4^{\circ} \mathrm{C}$, then rinsed in PBS, and incubated with Alexa Fluor $^{\circledR}$ 488-labeled anti-rabbit IgG (A11034, Molecular Probes; $\times 1000$ ) for $1 \mathrm{~h}$ at room temperature. They were then rinsed in PBS and stained with 4',6-diamidino-2-phenylindole to visualize the nuclei. To prepare negative control samples, the above-mentioned procedures were carried out using normal rabbit serum instead of anti-sclerostin as the primary antibody. A fluorescence microscope (BX51; Olympus, Tokyo, Japan) was used to observe the samples.

Transmission electron microscopy. The regenerating scales were fixed in $4 \%$ PFA and $4 \%$ glutaraldehyde in $0.1 \mathrm{M}$ phosphate buffer at $4^{\circ} \mathrm{C}$ overnight. The samples were rinsed thoroughly in $0.1 \mathrm{M}$ phosphate buffer, post-fixed in 1\% osmium tetroxide (Merck $\mathrm{KGaA}$, Darmstadt, Germany) for $1 \mathrm{~h}$ at $4^{\circ} \mathrm{C}$, and dehydrated in graded ethanol. After 5 min treatment with propylene oxide, the samples were infiltrated with Epon 812 (TAAB Laboratories, Berks, U.K.), to which accelerator DMP-30 was added. The samples were then cured at $60^{\circ} \mathrm{C}$ for 2 days. Ultrathin sections perpendicular to the scale surface were cut using a diamond knife. Sections were then stained with aqueous lead citrate. Some of the samples were decalcified with 5\% EDTA for 4 days after fixation, then treated as described above. The ultrathin sections obtained from the decalcified samples were stained with $1 \%$ tannic acid, alcoholic uranyl acetate and aqueous lead citrate. All ultrathin sections were observed under a transmission electron microscope (H-7100; Hitachi, Tokyo, Japan) at a $100 \mathrm{kV}$ accelerating voltage.

Space experiment on the International Space Station. Goldfish were anesthetized with $0.03 \%$ ethyl 3-aminobenzoate methanesulfonic acid salt (Sigma-Aldrich Inc.; neutralized using $\mathrm{NaHCO}_{3}$ ), and scales were removed one by one using sharpened forceps, as shown in Suzuki et al. (2009). Subsequently, goldfish were maintained at $26^{\circ} \mathrm{C}$. On day 12 after scale removal, goldfish were bred in water with the anti-infection reagent Green F Gold (Japan Pet Design Co. Ltd., Tokyo, Japan). On the following day, a few regenerating scales were collected from each goldfish. The scales with similar cell activities were selected as previously described (Ikegame et al. 2019). Subsequently, the regenerating scales were sampled on day 14 from goldfish on ice that were sterilized using hypochlorous and fungizone solutions and immersed in Leibovitz's L-15 medium containing $10 \% \mathrm{FCS}, 100 \mathrm{U} / \mathrm{mL}$ penicillin, $100 \mu \mathrm{g} /$ $\mathrm{mL}$ streptomycin, and $200 \mu \mathrm{g} / \mathrm{mL}$ kanamycin. Thereafter, the sterilized scales were packed into a culture chamber (Cell Experiment Small Chamber; Chiyoda Corporation, Yokohama, Japan). The culture chambers were kept at $2.5-4.0^{\circ} \mathrm{C}$ and sent to the International Space Station (ISS) by Space Shuttle Atlantis (STS-132) (Yano 2011). After their arrival on the ISS, they were incubated for $86 \mathrm{~h}$ under microgravity at the Cell Biology Experiment Facility (CBEF) (Yano 2011). The CBEF is equipped with a centrifuge to treat samples with one gravity [in-flight artificial microgravity (F-1g)] (Yano et al. 2012). This facility has the capability of culturing bio-specimens at a temperature range of $15-40^{\circ} \mathrm{C}$ (Yano et al. 2012). The differences among the sensors ranged from $0.1^{\circ} \mathrm{C}$ to $0.2^{\circ} \mathrm{C}$ (Yano et al. 2012). During incubation in the $\mathrm{CBEF}$, culture chambers installed in the microgravity section $(\mathrm{F}-\mu \mathrm{g})$ and $1 \mathrm{G}$ section (F$1 \mathrm{~g})$ of the CBEF were incubated at $21.9-22.0^{\circ} \mathrm{C}$ in the Measurement Experiment Unit (MEU) (Yano 
Table 1 Primer sequences used for quantitative real-time PCR

\begin{tabular}{cll}
\hline Target gene & \multicolumn{1}{c}{ Forward sequence } & \multicolumn{1}{c}{ Reverse sequence } \\
\hline Sost & 5'-CCGATTACCGCTGCATTCCT-3' & 5'-CGTCCGATTGGTTGTGATGG-3' \\
Dkk1 & 5'-AAGCACAAGAGGAAAGGCACCCAT-3' & 5'- TTAGTGTCTCTGGCAAGTGTGCAG-3' \\
Wifl & 5'-TGCCGAAGAGCAAGGAAGC-3' & 5'-TCCTGAAGTCGTGTGTGAATGGGG-3' \\
Efla & 5'-ATTGTTGCTGGTGGTGTTGG-3' & 5'-GGCACTGACTTCCTTGGTGA-3' \\
\hline
\end{tabular}

2011, 2012). The culture chambers were treated with $1 \mathrm{~g}$ gravity in the $1 \mathrm{G}$ section, whereas they were kept without the gravity treatment in the microgravity section. After incubation, culture chambers were removed from the MEU. Then, the culture medium in the culture chamber was replaced with RNAlater (Sigma-Aldrich, St. Louis, MO, USA) for gene expression analysis. Scales treated with RNAlater were stored at $-96^{\circ} \mathrm{C}$ until the return of STS-132 to the Kennedy Space Center in Florida.

Quantitative real-time PCR. Isolation of total RNA and cDNA synthesis were performed using kits (Qiagen GmbH, Hilden, Germany) in accordance with the manufacturer's instructions (Ishizu et al. 2018). Each quantitative real-time PCR reaction was performed using real-time PCR apparatus (Mx3000 $\mathrm{p}^{\mathrm{TM}}$; Stratagene, La Jolla, CA, USA). For the PCR reaction, a cDNA template mixed with the appropriate primers was combined with SYBR Premix Ex Taq (Takara Bio Inc., Shiga, Japan) (Ikegame et al. 2019). The elongation factor $1 \alpha(E f l \alpha)$ gene was used for normalization. PCR primer sequences used in the current study are listed in Table 1.

Statistics. All results are expressed as means \pm SEM $(n=4)$. The values of the control scales were compared with those of the experimental scales. Data were assessed using Student's $t$-tests, and the significance level chosen was $P<0.05$.

\section{RESULTS AND DISCUSSION}

Detection of cells expressing $m R N A$ of Sost (sclerostin) and its protein in the regenerating scales of goldfish

To obtain insight into whether the cells in the fish scales expressed the mRNA of Sost, whole-mount in situ hybridization (ISH) was conducted using the regenerating scales of the goldfish (Figs. 1 and 2). First, we focused on the peripheral area of the scales, where the ridges as well as the grooves exist (Fig. 1A). The ISH with the antisense Sost probe detected the signals in numerous cells located along the ridges and in a few cells in the grooves
(Fig. 1B), whereas that with the sense Sost probe did not (Fig. 1C), confirming their specificity. We next performed immunohistochemistry (IHC) with the sclerostin antibody. As shown in Figure 1D, IHC detected the signals in cells that were located mainly along the inner slope of the ridges and in a few cells in grooves, which were not detected by IHC with normal IgG (Fig. 1E). Taken together, we found that cells located in grooves and along ridges express the mRNA of Sost (sclerostin) and its protein in the regenerating scales of goldfish.

Next, we focused on the central area of the scales, where the grooves form a mesh-like structure (Fig. 2A). The ISH with the antisense Sost probe detected signals in cells located in the grooves (Fig. 2B), whereas that with the sense Sost probe did not (Fig. 2C). We next performed IHC with the sclerostin antibody to evaluate whether cells containing the sclerostin protein exist in the scales. As shown in Figure 2D, IHC detected signals in cells located in the grooves, which were not detected by IHC with normal IgG (Fig. 2E). IHC signals were also detected in cells outside the groove (Fig. 2D), although Sost signals of ISH were very weak in them. The reason why the different expression profiles exist between Sost mRNA and sclerostin protein have not been identified. A possible explanation is the different half-life between Sost mRNA and sclerostin protein. In future study, we will test this possibility by evaluating the time-dependent expression of Sost mRNA and sclerostin protein during the regeneration of scales.

It should be noted that several types of cells that regulate bone metabolism, such as osteoclasts and osteoblasts, were reported to exist in grooves and ridges of the regenerating scales of goldfish (Yoshikubo et al. 2005; Suzuki et al. 2007, 2008b; Yachiguchi et al. 2014; Ikegame et al. 2019; Yamamoto et al. 2020). Accordingly, we further examined the sclerostin-producing cells using cryosections in addition to whole-mount analysis, as described below. 

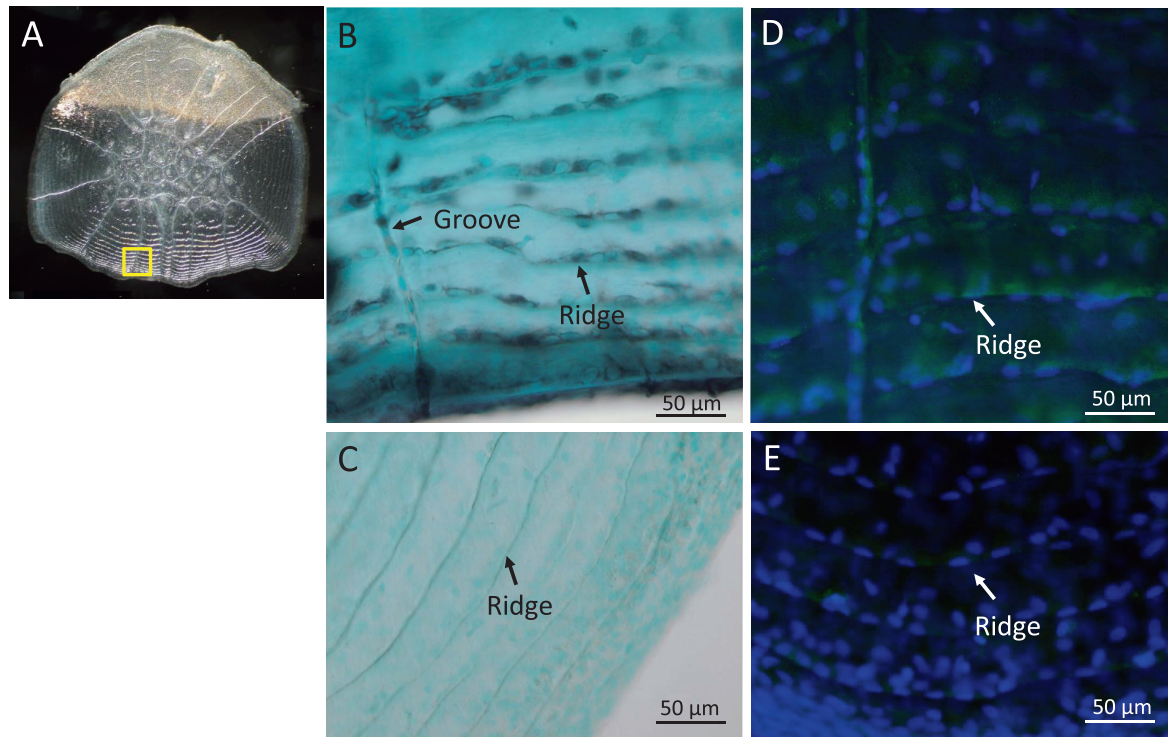

Fig. 1 Whole-mount detection of Sost/sclerostin in the peripheral area of the regenerating scales by in situ hybridization (ISH) and immunohistochemistry (IHC). A: Binocular view of regenerating scale on day 15. The parts corresponding to the area in the yellow square are magnified and shown in B to E. B: Detection of Sost mRNA with an antisense probe by ISH. Positive staining is indicated by a dark purple color. C: Negative control for ISH with Sost sense probe. D: Detection of sclerostin with anti-sclerostin antibody by IHC. Positive staining is indicated by a green color. E: Negative control for IHC with normal rabbit IgG. Arrows indicate grooves or ridges. Nuclei were stained with methyl green in B and C (light green) and with 4',6-diamidino-2-phenylindole (DAPI) in D and E (blue). The images are representative data obtained from repeated experiments using five or more scales from different goldfish. The images were taken from the epithelial side (osseous layer) of scale.
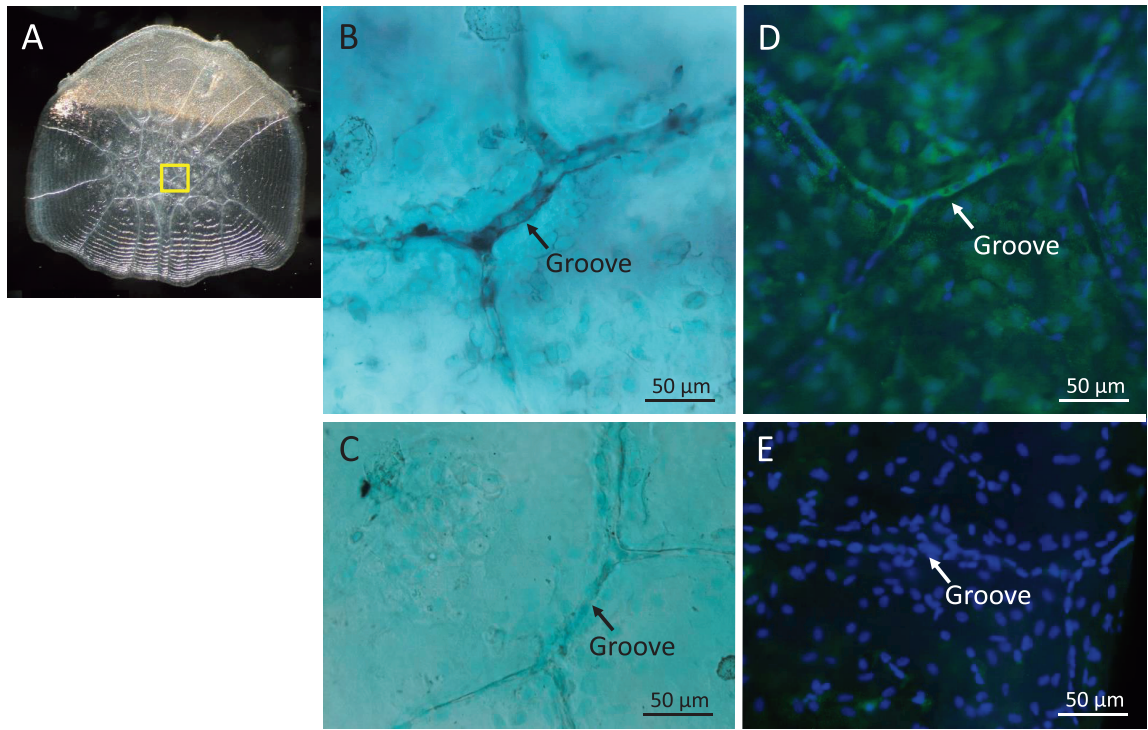

Fig. 2 Whole-mount detection of Sost/sclerostin in the central area of the regenerating scales by in situ hybridization (ISH) and immunohistochemistry $(\mathrm{IHC})$. A: Binocular view of regenerating scale on day 15 . The parts corresponding to the area in the yellow square are magnified and shown in B to E. B: Detection of Sost mRNA with an antisense probe by ISH. Positive staining is indicated by a dark purple color, which is observed in the cytoplasm of 6 to 8 mononuclear cells in the groove. C: Negative control for ISH with Sost sense probe. D: Detection of sclerostin with anti-sclerostin antibody by IHC. Positive staining is indicated by a green color. E: Negative control for IHC with normal rabbit IgG. Arrows indicate grooves. Nuclei were stained with methyl green in B and C (light green) and with DAPI in D and E (blue). The images are representative data obtained from repeated experiments using five or more scales from different goldfish. The images were taken from the epithelial side (osseous layer) of scale. 

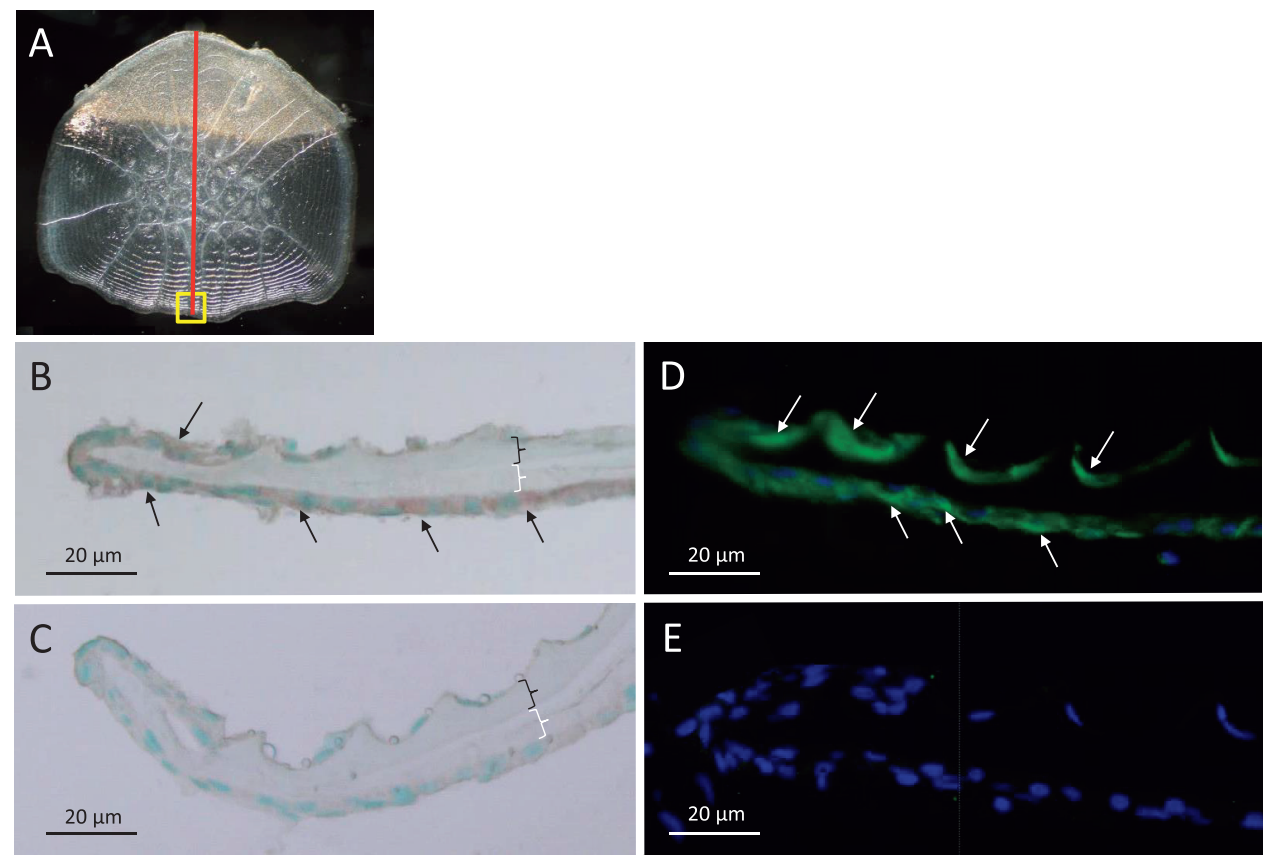

Fig. 3 Detection of Sost/sclerostin in the peripheral area of the regenerating scales with cryosections by in situ hybridization (ISH) and immunohistochemistry (IHC). A: Binocular view of a regenerating scale on day 15. The parts corresponding to the area in the yellow square in cryosections are magnified and shown in B to $\mathrm{E}$, in which the epidermis and dermis sides are shown on the upper and lower sides, respectively. The cryosections were cut along the red line. B: Detection of Sost mRNA with an antisense probe by ISH. Positive staining is indicated by a brownish-purple color (arrows). Osseous and fibrous layers are indicated by black and white curly braces respectively. C: Negative control for ISH with a Sost sense probe. Osseous and fibrous layers are indicated by black and white curly braces respectively. D: Detection of sclerostin with anti-sclerostin antibodies by IHC. Positive staining is indicated by a green color (arrows). E: Negative control for IHC with normal rabbit IgG. Nuclei were stained with methyl green in B and C (light green) and with DAPI in D and E (blue). The images are representative data obtained from repeated experiments using five or more scales from different goldfish.

Cells expressing sclerostin are half-embedded cells, bone lining cells and osteoblasts

To evaluate the distribution of cells expressing Sost mRNA separately in the osseous and fibrous layers of the scales, ISH was conducted using cryosections of the regenerating scales (Figs. $3 \mathrm{~A}$ and $4 \mathrm{~A}$ ). In both the central and peripheral areas of the scales (Figs. 3B and 4C), ISH with the antisense Sost probe detected signals in the cells covering the fibrous layers, but not in the cells of the osseous layers except for a few cells close to the peripheral end of the scale (Fig. 3B). This result is inconsistent with our finding that the signals were detected in the cells on the osseous layers of grooves and along ridges by whole mount ISH (Figs. 1 and 2). We speculate that the ISH signals in the cells of the osseous layers are too weak to be detected on the thin cryosections. The specificity of the signals was confirmed by ISH with the sense Sost probe, which did not detect the signals (Figs. 3C and 4D). We next performed IHC with the sclerostin antibody (Figs. 3D and 4E), whose specificity was confirmed by IHC with nor- mal IgG (Figs. 3E and 4F). The distribution of cells containing sclerostin protein was similar to that of cells expressing Sost mRNA covering the fibrous layers. IHC signals were also detected in cells of the osseous layers. Flat lining cells and half-embedded cells in the mineralized matrix expressed sclerostin (Figs. 3D and 4E).

Of note, cells with ISH or IHC signals on fibrous layers were found to have rounded shapes, suggesting that they are producing the matrix of scale (Yoshikubo et al. 2005; Suzuki et al. 2007, 2008b; Ikegame et al. 2019; Yamamoto et al. 2020). In addition to round cells, ISH or IHC signals were detected in flat cells lining the osseous layer along peripheral ridges and on the mineralized matrix surface in the center (Figs. 1D, 2B, 2D, 3D and 4E). The cell shape and situation correspond to inactive osteoblasts-lining cells in bone tissue. Therefore, our results indicate that osteoblasts in scales express Sost mRNA.

In addition, Sost/sclerostin signals were detected in half-embedded cells in a groove or in the calci- 

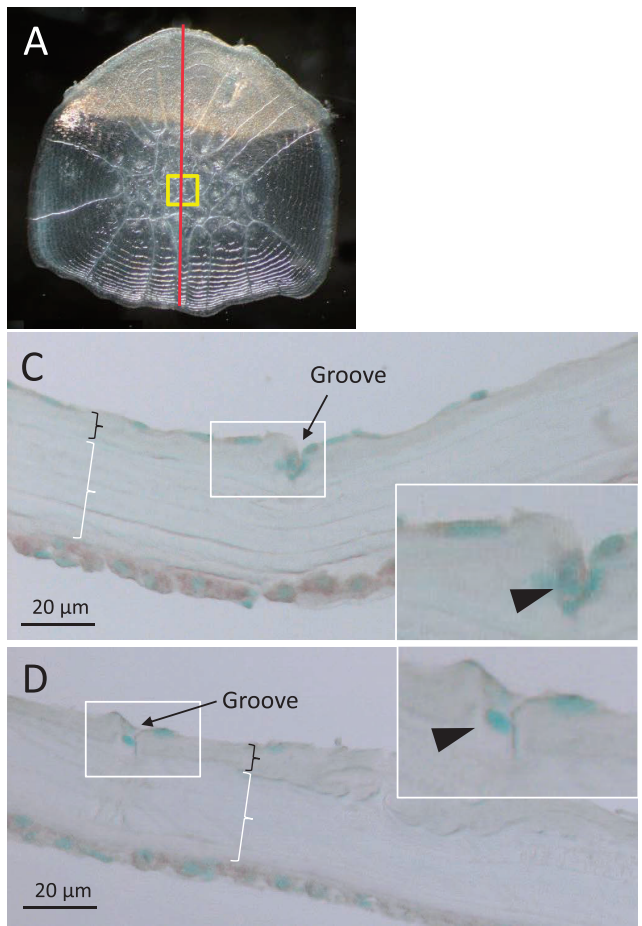
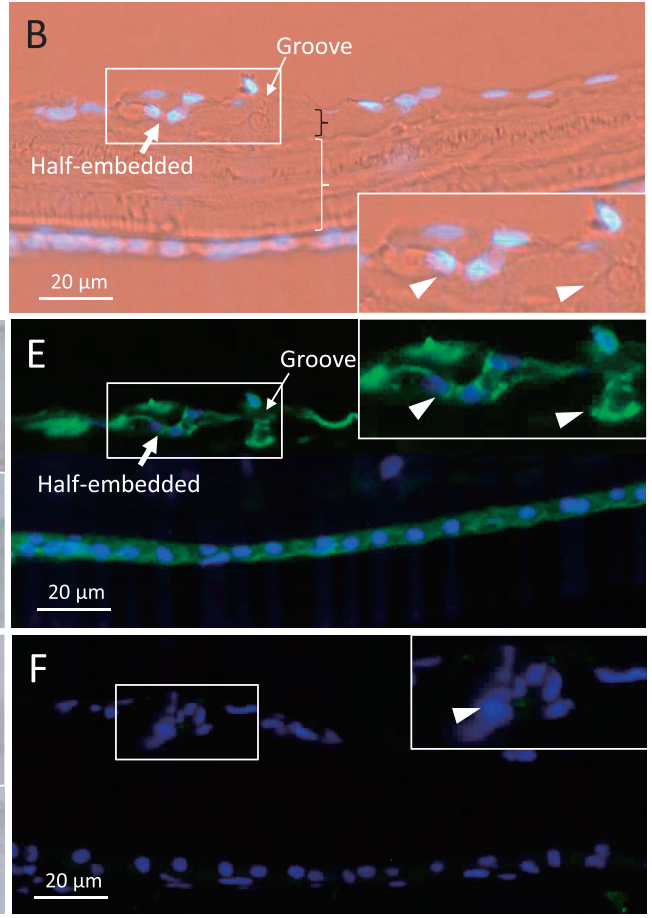

Fig. 4 Detection of Sost/Sclerostin in the central area of regenerating scales with cryosections by in situ hybridization (ISH) and immunohistochemistry (IHC). A: Binocular view of a regenerating scale on day 15. The parts corresponding to the area in the yellow square are magnified in cryosections and shown in B to F. The cryosections were cut along the red line. B: Bright-field observation of the cryosection. The upper side is the epidermis side and is composed of a thin osseous layer covered with flat lining cells. Some cells were half embedded in the calcified matrix. The lower side is the dermis side and is composed of a thick fibrous layer covered with scale osteoblasts. The inset is a magnified view of the area in the square. The arrowheads indicate half-embedded cells in a groove or in the calcified matrix. Osseous and fibrous layers are indicated by black and white curly braces respectively. C: Detection of Sost mRNA with an antisense probe by ISH. Positive staining is indicated by a brownish-purple color. The inset is a magnified view of the area in the square. The arrowhead indicates half-embedded cells in a groove. Osseous and fibrous layers are indicated by black and white curly braces respectively. D: Negative control for ISH with a Sost sense probe. The inset is a magnified view of the area in the square. The arrowhead indicates half-embedded cells in a groove. Osseous and fibrous layers are indicated by black and white curly braces respectively. E: Same field of view as B, observed with fluorescent microscopy. Sclerostin was detected with anti-sclerostin antibodies by IHC. Positive staining is indicated by a green color. The inset is a magnified view of the area in the square. The arrowheads indicate half-embedded cells in a groove or in the calcified matrix. $\mathbf{F}$ : Negative control for IHC with normal rabbit IgG. The inset is a magnified view of the area in the square. The arrowhead indicates half-embedded cells in a groove or in the calcified matrix. Nuclei were stained with methyl green in $C$ and $D$ (light green) and with DAPI in B (pale blue), E (blue), and F (blue). The nuclei appear pale blue in B due to the fact that the blue fluorescent color of DAPI and the visible light image are superimposed. The images are representative data obtained from repeated experiments using five or more scales from different goldfish.

fied matrix (Fig. 4C, E). Judging from observation by transmission electron microscopy, we found two types of half-embedded cells in the osseous layer. One type was observed at the bottom of the groove, in contact with the collagen bundles of the fibrous layer (Fig. 5A). The other type was almost surrounded by the mineralized matrix (Fig. 5B). Thus, we speculate that these half-embedded cells are osteocyte-like cells, which terminally differentiate from osteoblasts and become embedded in the bone matrix (Sawa et al. 2019).

The basis for this speculation is not only the cell localization (half embedded in the mineralized matrix) and Sost/sclerostin expression. Osteoclasts expressing tartrate-resistant acid phopshatate have been reported to exist in the osseous layers, especially along grooves, but not in fibrous layers (Ikegame et al. 2019). In addition, RANKL expression has been found mainly in cells in the grooves (Yamamoto et al. 2020). Accordingly, it is speculated that half-embedded osteocyte-like cells in the osseous layers promote osteoclast differentiation and activation by producing RANKL (Yamamoto et al. 2020), as those cells do in mammalian bone (Nakashima et al. 


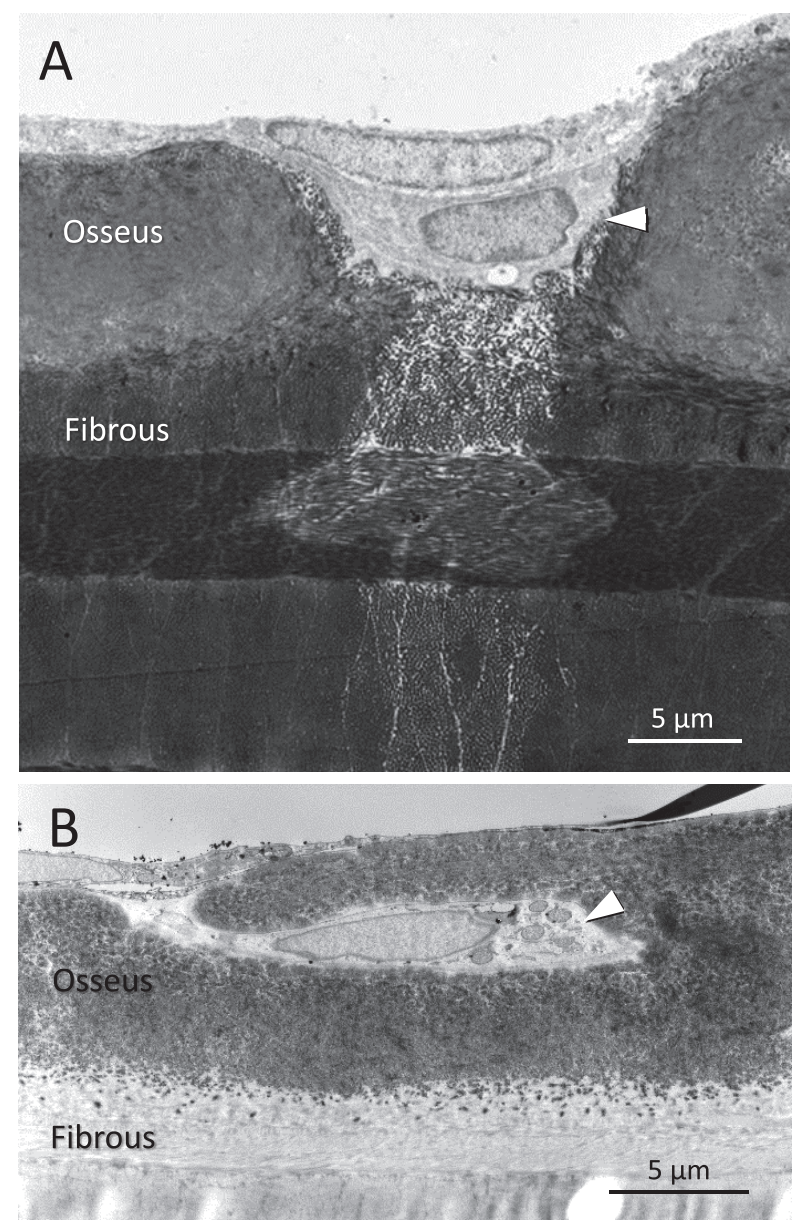

Fig. 5 Electron microscopic observation of the half-embedded cells in osseous layer of regenerating scale. A: A decalcified scale. A half-embedded cell (arrowhead) in a groove observed between the mineralized matrices. A part of fibrous layer is shown in the bottom half of the panel; plywood-like collagen fibers are stained by tannic acid. B: An un-decalcified scale. A half-embedded cell (arrowhead) observed in the mineralized matrix.

\section{1).}

Increased Sost expression in the regenerating scales of goldfish under microgravity in outer space

Our previous experiments in space aboard the ISS demonstrated that osteoclast activity along grooves and the ratio of RANKL : OPG expression in goldfish scale increase under microgravity conditions (Ikegame et al. 2019). It has been reported that sclerostin simulates RANKL production in osteocytes (Wijenayaka et al. 2011) and inhibits OPG production in osteoblasts/osteocytes by disturbing the Wnt signaling pathway to activate osteoclasts (Kubota et al. 2009; Kobayashi et al. 2016; Delgado-Calle et al. 2017; Figurek et al. 2020). This fact, together with our finding of the presence of Sost-expressing cells in goldfish scales, indicates that sclerostin may contribute to the microgravity-induced activation of osteoclasts in goldfish scales. We compared the expression levels of Sost, as well as genes encoding suppressors of the Wnt signaling pathway, Dkkl and Wifl, in goldfish scales kept under microgravity conditions in outer space $(\mathrm{F}-\mu \mathrm{g})$ with those of goldfish scales treated with $1 \mathrm{~g}$ gravity in outer space $(\mathrm{F}-1 \mathrm{~g})$. Microgravity was found to increase the expression of Sost, whereas it did not have any effect on $D k k 1$ or Wif1 (Fig. 6).

Sost/sclerostin expression is known to be upregulated by mechanical unloading and plays a critical role in bone mechano-response (Robling et al. 2008; Lin et al. 2009). Our results showed for the first time that the expression of Sost in fish scales is stimulated by microgravity. In support of our idea, it was reported that the Sost expression level in mouse calvariae was significantly higher than that of ground control after 30 days of spaceflight (Macaulay et al. 2017). These suggest a mechanism underlying the osteoporosis-like loss of bone mass in outer space: microgravity increases sclerostin in osteocytes and osteoblasts by facilitating the expression of the Sost gene. The increased sclerostin then excessively activates osteoclasts, leading to the loss of bone mass.

\section{Acknowledgments}

This study was supported in part by grants to N.S. (Grant-in-Aid for Scientific Research [C] No. 20K06718 by JSPS; Grant-in-Aid for Space Utilization by the Japan Aerospace Exploration Agency), to T.S. (Grant-in-Aid for Scientific Research [C] No. $18 \mathrm{~K} 06312$ by JSPS), to Y.T. (Grant-in-Aid for Scientific Research [C] No. 20K12619 by JSPS), to J.H. (Grant-in-Aid for Scientific Research [B] No. $20 \mathrm{H} 04565$ and [C] No. 18KT0068 by JSPS), and to A.H. (Grant-in-Aid for Scientific Research [C] No. 20K06718 by JSPS). In addition, this study was performed as part of a cooperative research program of the Institute of Nature and Environmental Technology, Kanazawa University, Accept Nos. 2020, 2030, 2032, and 2033. The authors thank Ms. Leticia Shinotsuka, University of Sao Paulo, for help preparing cryosections and ISH.

\section{CONFLICT OF INTERESTS}

The authors declare that they have no conflicts of interest. 

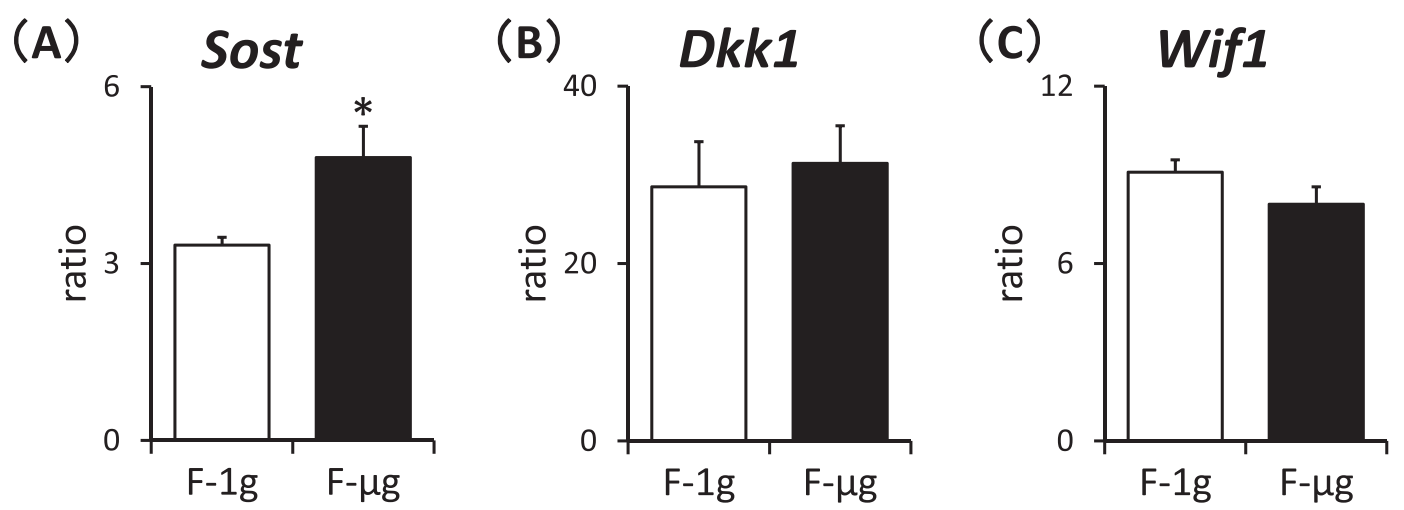

Fig. 6 Expression analyses of Wnt inhibitors Sost, Dkk1, and Wif1 in the regenerating scales of goldfish under microgravity by quantitative RT-PCR. Ef1a gene was used for normalization of respective gene expression. ${ }^{*} P<0.05$ versus control; $n=4$

\section{REFERENCES}

Azuma K, Kobayashi M, Nakamura M, Suzuki N, Yashima S, et al. (2007) Two osteoclastic markers expressed in multinucleate osteoclasts of goldfish scales. Biochem Biophys Res Commun 362, 594-600.

Balemans W, Ebeling M, Patel N, Van Hul E, Olson P, et al. (2001) Increased bone density in sclerosteosis is due to the deficiency of a novel secreted protein (SOST). Hum Mol Genet 10, 537-543.

Balemans W, Patel N, Ebeling M, Van Hul E, Wuyts W, et al. (2002) Identification of a $52 \mathrm{~kb}$ deletion downstream of the SOST gene in patients with van Buchem disease. $J$ Med Genet 39, 91-97.

Bereiter-Hahn J and Zylberberg L (1993) Regeneration of teleost fish scale. Comp Biochem Physiol Part A 105, 625-641.

Brunkow ME, Gardner JC, Van Ness J, Paeper BW, Kovacevich $\mathrm{BR}$, et al. (2001) Bone dysplasia sclerosteosis results from loss of the SOST gene product, a novel cystine knot-containing protein. Am J Hum Genet 68, 577-589.

Collette NM, Yee CS, Murugesh D, Sebastian A, Taher L, et al. (2013) Sost and its paralog Sostdc1 coordinate digit number in a Gli3-dependent manner. Dev Biol 383, 90-105.

Delgado-Calle J, Sato AY and Bellido T (2017) Role and mechanism of action of sclerostin in bone. Bone 96, 29-37.

de Vrieze E, Moren M, Metz JR, Flik G and Lie KK (2014) Arachidonic acid enhances turnover of the dermal skeleton: studies on zebrafish scales. PLoS One 9, e89347.

Ellies DL, Viviano B, McCarthy J, Rey JP, Itasaki N, et al. (2006) Bone density ligand, sclerostin, directly interacts with LRP5 but not $\mathrm{LRP}^{\mathrm{G} 171 \mathrm{~V}}$ to modulate Wnt activity. $J$ Bone Miner Res 21, 1738-1749.

Figurek A, Rroji M and Spasovski G (2020) Sclerostin: a new biomarker of CKD-MBD. Int Urol Nephrol 52, 107-113.

Hanmoto T, Tabuchi Y, Ikegame M, Kondo T, Kitamura K, et al. (2017) Effects of low-intensity pulsed ultrasound on osteoclasts: analysis with goldfish scales as a model of bone. Biomed Res (Tokyo) 38, 71-77.

Henry JP and Bordoni B (2020) Histology, Osteoblasts. In StatPearls. StatPearls Publishing, Treasure Island, FL.

Hinton PV, Rackard SM and Kennedy OD (2018) In vivo osteocyte mechanotransduction: recent developments and future directions. Curr Osteoporos Rep 16, 746-753.

Ikegame M, Hattori A, Tabata MJ, Kitamura K, Tabuchi Y, et al. (2019) Melatonin is a potential drug for the prevention of bone loss during space flight. J Pineal Res 67, e12594.

Ishizu H, Sekiguchi T, Ikari T, Kitamura KI, Kitani Y, et al. (2018) alpha-Melanocyte-stimulating hormone promotes bone resorption resulting from increased osteoblastic and osteoclastic activities in goldfish. Gen Comp Endocrinol 262, 99-105.

Kearns AE, Khosla S and Kostenuik PJ (2008) Receptor activator of nuclear factor kappaB ligand and osteoprotegerin regulation of bone remodeling in health and disease. Endocr Rev 29, 155-192.

Kitamura K, Suzuki N, Sato Y, Nemoto T, Ikegame M, et al. (2010) Osteoblast activity in the goldfish scale responds sensitively to mechanical stress. Comp Biochem Physiol Part A 156, 357-363.

Kobayashi Y, Uehara S, Udagawa N and Takahashi N (2016) Regulation of bone metabolism by Wnt signals. $J$ Biochem 159, 387-392

Kondo Y, Irie K, Ikegame M, Ejiri S, Hanada K, et al. (2001) Role of stromal cells in osteoclast differentiation in bone marrow. J Bone Miner Metab 19, 352-358.

Kubota T, Michigami T and Ozono K (2009) Wnt Signaling in bone metabolism. J Bone Miner Metab 27, 265-271.

Lacey DL, Boyle WJ, Simonet WS, Kostenuik PJ, Dougall WC, et al. (2012) Bench to bedside: elucidation of the OPGRANK-RANKL pathway and the development of denosumab. Nat Rev Drug Discov 11, 401-419.

Li X, Zhang Y, Kang H, Liu W, Liu P, et al. (2005) Sclerostin binds to LRP5/6 and antagonizes canonical Wnt signaling. $J$ Biol Chem 280, 19883-19887.

Lin C, Jiang X, Dai Z, Guo X, Weng T, et al. (2009) Sclerostin mediates bone response to mechanical unloading through antagonizing Wnt/ $\beta$-catenin signaling. J Bone Miner Res 24, $1651-1661$.

Macaulay TR, Siamwala JH, Hargens AR, Macias BR, et al. (2017) Thirty days of spaceflight does not alter murine calvariae structure despite increased Sost expression. Bone Rep 7, 57-62.

Nakashima T, Hayashi M, Fukunaga T, Kurata K, Oh-Hora M, et al. (2011) Evidence for osteocyte regulation of bone homeostasis through RANKL expression. Nat Med 17, 1231-1234.

Ohira Y, Shimizu M, Ura K and Takagi Y (2007) Scale regeneration and calcification in goldfish Carassius auratus: quantitative and morphological processes. Fisheries Sci 73, 46-54.

Pajevic PD, Spatz JM, Garr J, Adamson C and Misener L (2013) 
Osteocyte biology and space flight. Curr Biotechnol 2, 179183.

Poole KE, van Bezooijen RL, Loveridge N, Hamersma H, Papapoulos SE, et al. (2005) Sclerostin is a delayed secreted product of osteocytes that inhibits bone formation. FASEB $J$ 19, 1842-1844.

Robling AG, Niziolek PJ, Baldridge LA, Condon KW, Allen MR, et al. (2008) Mechanical stimulation of bone in vivo reduces osteocyte expression of Sost/sclerostin. J Biol Chem 283, $5866-5875$.

Sawa N, Fujimoto H, Sawa Y and Yamashita J (2019) Alternating differentiation and dedifferentiation between mature osteoblasts and osteocytes. Sci Rep 9, 13842.

Silva I and Branco JC (2011) RANK/RANKL/OPG: literature review. Acta Reumatol Port 36, 209-218.

Staehling-Hampton K, Proll S, Paeper BW, Zhao L, Charmley P, et al. (2002) A 52-kb deletion in the SOST-MEOX1 intergenic region on 17q12-q21 is associated with van Buchem disease in the Dutch population. Am J Med Genet 110, 144 152.

Suzuki N and Hattori A (2002) Melatonin suppresses osteoclastic and osteoblastic activities in the scales of goldfish. $J$ Pineal Res 33, 253-258.

Suzuki N, Hanmoto T, Yano S, Furusawa Y, Ikegame M, et al. (2016) Low-intensity pulsed ultrasound induces apoptosis in osteoclasts: fish scales are a suitable model for analysis of bone metabolism by ultrasound. Comp Biochem Physiol Part A 195, 26-31.

Suzuki N, Kitamura K, Omori K, Nemoto T, Satoh Y, et al. (2009) Response of osteoblasts and osteoclasts in regenerating scales to gravity loading. Biol Sci Space 23, 211-217.

Suzuki N, Kitamura K, Nemoto T, Shimizu N, Wada S, et al. (2007) Effect of vibration on osteoblastic and osteoclastic activities: analysis of bone metabolism using goldfish scale as a model for bone. Adv Space Res 40, 1711-1721.

Suzuki N, Omori K, Nakamura M, Tabata MJ, Ikegame M, et al. (2008a) Scale osteoblasts and osteoclasts sensitively respond to low-gravity loading by centrifuge. Biol Sci Space 22, 3-7.

Suzuki N, Somei M, Seki A, Reiter RJ and Hattori A (2008b) Novel bromomelatonin derivatives as potentially effective drugs to treat bone diseases. J Pineal Res 45, 229-234.

Suzuki N, Suzuki T and Kurokawa T (2000) Suppression of osteoclastic activities by calcitonin in the scales of goldfish (freshwater teleost) and nibbler fish (seawater teleost). Peptides 21, 115-124.

Tatsumi S, Ishii K, Amizuka N, Li M, Kobayashi T, et al. (2007) Targeted ablation of osteocytes induces osteoporosis with defective mechanotransduction. Cell Metab 5, 464-475.

Wijenayaka AR, Kogawa M, Lim HP, Bonewald LF, Findlay DM, et al. (2011) Sclerostin stimulates osteocyte support of osteoclast activity by a RANKL-dependent pathway. PLOS One 6, e25900.

Yachiguchi K, Sekiguchi T, Nakano M, Hattori A, Yamamoto M, et al (2014) Effect of inorganic mercury and methylmercury on osteoclasts and osteoblasts in the scales of the marine teleost as a model system of bone. Zool Sci 31, 330-337.

Yamamoto T, Ikegame M, Kawago U, Tabuchi Y, Hirayama J, et al. (2020) Detection of RANKL-producing cells and osteoclastic activation by the addition of exogenous RANKL in the regenerating scales of goldfish. Biol Sci Space 34, 34 40 .

Yano S (2011) Preparation and overview of fish scales experiment. Space Utiliz Res 27, 213-216.

Yano S, Masuda D, Kasahara H, Omori K, Higashibata A, et al. (2012) Excellent thermal control ability of cell biology experiment facility (CBEF) for ground-based experiments and experiments onboard the Kibo Japanese experiment module of international space station. Biol Sci Space 26, 12-20.

Yoshikubo H, Suzuki N, Takemura K, Hoso M, Yashima S, et al. (2005) Osteoblastic activity and estrogenic response in the regenerating scale of goldfish, a good model of osteogenesis. Life Sci 76, 2699-2709. 